\title{
Diversion of retinal blood flow by photocoagulation
}

\author{
D. W. HILL AND S. YOUNG \\ From the Research Department of Ophthalmology, Royal College of Surgeons of England, \\ Lincoln's Inn Fields, London
}

SUMMARY The normal distribution of the retinal blood flow in the cat eye was modified by photocoagulation to part of the territory supplied by a major arteriole, or by occluding a branch. Volume inflow to the treated territory was reduced, and there was also a reduction of linear flow and, to a less extent, calibre in the parent vessel. Branches of the parent vessel supplying untreated territory showed marginal increases of volume inflow. An autoregulatory effect appeared to be operative. The relevance of these findings to clinical panretinal photocoagulation is discussed.

Photocoagulation has been widely employed in the treatment of retinovascular disease to ablate specific vascular lesions. Meyer-Schwickerath and Schott (1968) suggested that it might have a beneficial effect on the residual circulation; so the present work was undertaken to assess the redistribution of the retinal circulation in the healthy cat eye, following photocoagulation. A knowledge of the regional distribution of the retinal circulation had been acquired by previous work (Hill, 1977).

\section{Methods}

Domestic cats, preferably male, unselected save for weight exceeding $2.5 \mathrm{~kg}$ and freedom from overt disease, were anaesthetised on 2 occasions with pentobarbitone sodium by intravenous injection, $30 \mathrm{mg} / \mathrm{kg}$ body weight, supplemented by additional injections to maintain the level of anaesthesia when necessary. Respiration was controlled by a Palmer respiration pump with either intubation or tracheostomy. In the terminal experiments blood gas tensions were monitored and blood pressure recorded. Body temperature was monitored and kept as near to $39^{\circ} \mathrm{C}$ as possible.

The first, survival, experiment which concluded with photocoagulation to 1 eye, was followed after an interval of 21 to 49 days by the terminal experiment. After sacrifice the retinal circulation was injected with Indian ink; the eyes were removed and fixed; and the retinas were dissected out, flattened, and photographed (method, Goel, 1974).

Photocoagulation was applied either to the retinal

Address for reprints: Professor D. W. Hill, Royal College of Surgeons of England, 35-43 Lincoln's Inn Fields, London WC2A 3PN tissue between the vessels or to selected branch arterioles to produce occlusion. In the former case the applications covered an area of retina supplied by the branches of selected arterioles.

Retinal blood flow and arteriolar vessel calibre were assessed by high-speed cine angiography (Hill and Young, 1976), dye being injected into the infraorbital artery in the survival experiments and into the lingual artery in terminal experiments. Linear blood flow was estimated from the number of frames elapsing while the dye passed between 2 selected points on an arteriole, and calibre by densitometric measurement of the cine film or measurement of the projected cine image with a low-power microscope and a screw micrometer eyepiece.

Data were processed to provide estimates of linear flow at the retina $(\mathrm{mm} / \mathrm{s})$ and absolute calibre of the retinal vessels as revealed by their fluorescein image. Relative volume flow was derived from the product of linear flow and cross-sectional area, the crosssectional velocity profile being ignored.

In order to compare the blood flow in arterioles of different calibre and in different zones of the fundus, where a centrifugal reduction of flow has been demonstrated (Laatikainen, 1976), a corrected volume flow per unit area was computed. The area supplied by the arteriole concerned was adjusted to zone II of the retina (Goel, 1974) using the relative inflow values observed by Laatikainen (1976), zone I: II: III:: $1 \cdot 0: 0 \cdot 5: 0 \cdot 3$. This figure was divided in:o the calculated volume flow to produce a corrected volume flow per unit area $\left(\mathrm{V}_{\mathrm{aii}}\right)$.

In this study it was often desirable to compare the change at a given site between survival and terminal experiments. This was conveniently expressed in logarithmic units as the difference between the logarithm of the initial (survival) value and that of 


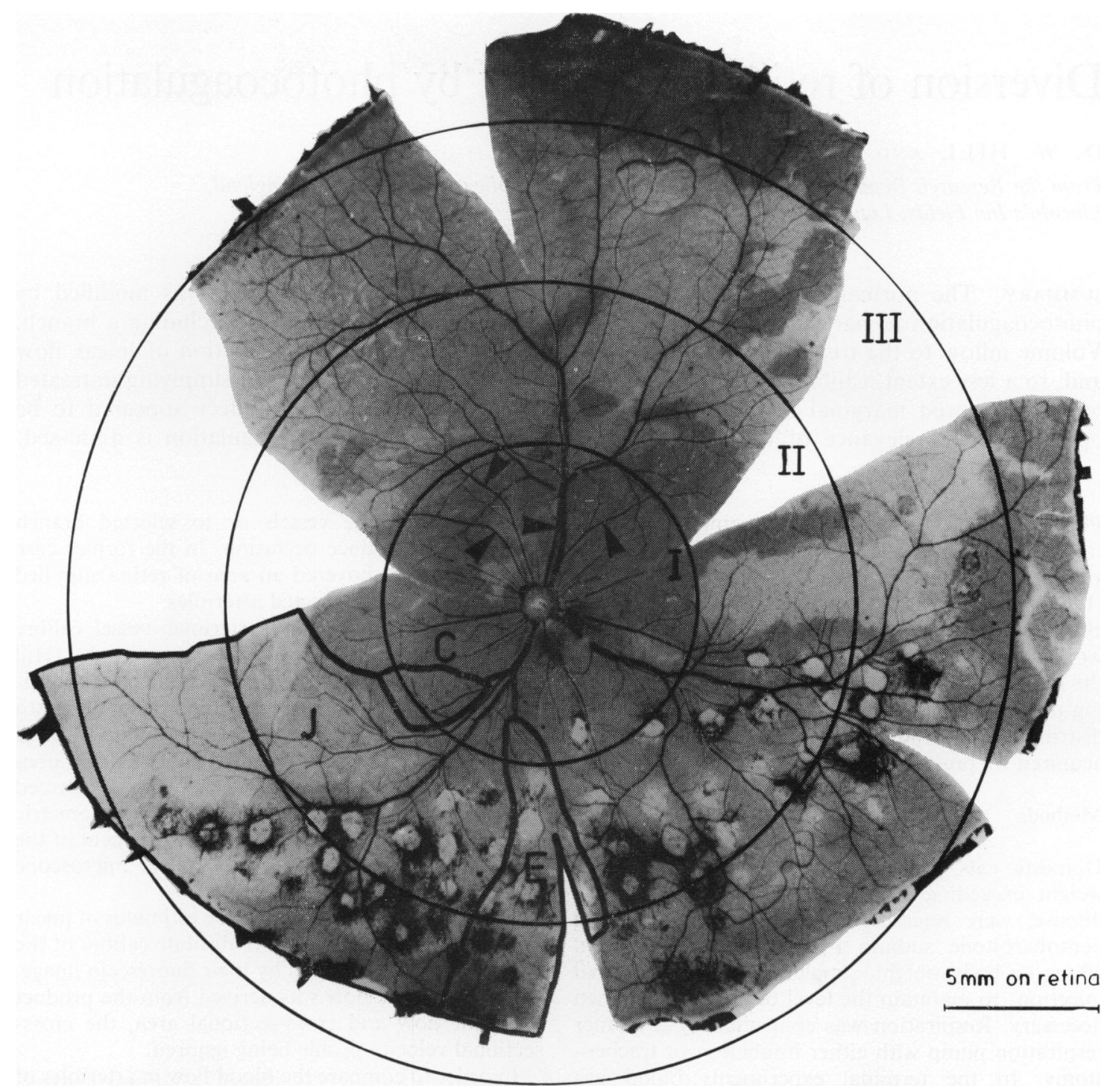

Fig. 1 Photograph of the injected retina of cat 115. Over the lower non-tapetal retina extensive diffuse photocoagulation has been applied. The areas supplied by stem, photocoagulation, and branch vessels have been labelled $\mathrm{J}, \mathrm{E}$, and $\mathrm{C}$, respectively. The arterioles concerned can be identified as they enter their areas at the apex close to the disc. Only some of the vessels assessed have been outlined, in order to avoid confusion. Above, the 4 superior vessels measured as controls are indicated by arrows. The zones of the retina are indicated by circles concentric with the optic disc; zone III also includes the retina outside the largest circle

the terminal value. On one occasion, the comparison of linear flow and calibre changes with area of photocoagulation, simple ratios were used to provide a more informative regression equation.

The retinal arterioles were considered in 3 groups - superior vessels, stem vessels, and branch arterioles. Superior vessels away from the site of photo- coagulation afforded control data. Stem vessels which supplied both photocoagulated and untreated retina were measured close to the point of origin of branch vessels which supplied untreated retina. A fourth category, photocoagulation vessels, comprised terminal branches of the stem vessels, supplying only coagulated tissue. Fig. 1 shows an 
example of the photocoagulated retina and the disposition of vessel categories.

\section{Results}

The experiments were carried out in two groups, 113-117, and 134-139. Owing to incomplete data only experiments $113,115,116,117,136$, and 139 are available for full analysis.

Flow into one or both lower quadrants of the retina in the treated eyes was disturbed by applying diffuse photocoagulation or by occluding an arteriole with photocoagulation. Table 1 summarises the treatment applied.

After processing the results 2 methods were adopted to test the effect of photocoagulation on the distribution of retinal blood flow; comparison of the changes in flow between initial and terminal experiments, in affected and control vessels, and comparison of the volume flow per unit area $\left(\mathrm{V}_{\mathrm{aii}}\right)$ at the terminal experiment in affected and control vessels.

\section{CONTROL VESSELS}

Comparison of the $\mathrm{V}_{\mathrm{aii}}$ in the superior arterioles of the two eyes of each animal, by the $t$ test for difference of means gave values of $t \leqslant 1.47$ with the corresponding probability, $\mathrm{P}<0 \cdot 1$. It was concluded that no significant differences existed between the pairs of eyes, so that the superior vessels of the treated eye could be used as control vessels for the effects of photocoagulation.

The changes, logarithmically expressed, between initial and final experiments, showed significant alterations in the linear flow rates and calibres of the control vessels. Applying the paired $t$ test to the results from all the superior vessels in all the experiments, a significant $(P<0.001)$ calibre change was found, with a mean value $-0.06 \log$ units (equivalent to a reduction to $87 \%$ of the initial value). Linear flow showed no bulk change (mean value $-0.01 \log$ units, equivalent to $98 \%$ of the initial value; $\mathbf{P}>0 \cdot 1$ ), but a significant variance ratio between as compared to within the groups of vessels in each eye $(\mathrm{F}, 3.90 ; \mathrm{P}, 0.05-0.01)$, indicating different flow changes in each eye. For each eye the mean changes in linear flow and calibre of the control vessels (Table 2) were added to the changes recorded in the vessels affected by photocoagulation to provide corrected values for comparison with the area of photocoagulation.

STEM AND PHOTOCOAGULATION VESSELS As a result of diffuse photocoagulation the area of healthy retina supplied by these vessels was reduced. Significant reductions were found in linear flow, calibre and $\mathrm{V}_{\mathrm{aii}}$, when tested by the paired $t$ test. The results are summarised in Table 3 . Although the photocoagulation vessels, as might be expected from their proximity to the treatment, were more affected, no significant difference could be demonstrated between their mean values and the stem mean values ( $t$ test, difference of means). A correlation was shown for the regression of both linear flow change $\left(\mathrm{r}_{3}, 0.88 ; \mathrm{P}, 0.05\right)$ and calibre change $\left(\mathrm{r}_{3}, 0.87 ; \mathrm{P}, 0.05\right)$ on the reduction of area of untreated retina supplied by stem vessels after photocoagulation. The regression equations $(y$, ratio of linear flow or calibre change, $x$, ratio of the retinal area) were $y=0 \cdot 19+1 \cdot 30 x$ and $y=0 \cdot 76+0 \cdot 35 x$, for linear flow and calibre change respectively.

\section{Table 1 Photocoagulation}

\begin{tabular}{lll}
\hline Expt. & Eye & Photocoagulation applied \\
\hline 113 & $\mathrm{R}$ & Inferior, diffuse, large \\
115 & $\mathrm{~L}$ & Inferior, diffuse, large \\
116 & $\mathrm{~L}$ & Infero-temporal, * confluent, large \\
117 & $\mathrm{R}$ & Infero-temporal, ${ }^{*}$ confluent, small \\
\hline 136 & $\mathrm{~L}$ & Nasal, distal arterial occlusion, partial \\
& & Temporal, diffuse, peripheral, small \\
\hline 139 & $\mathrm{R}$ & Nasal, proximal arterial occlusion, partial \\
& & Temporal, distal arterial occlusion \\
\hline
\end{tabular}

*Smaller areas with overlapping coagulations ( $c f$ Fig. 1)

Table 2 Correction factors, log units, derived from changes in superior vessels

\begin{tabular}{lllllll}
\hline $\begin{array}{l}\text { Experiment } \\
113\end{array}$ & 115 & 116 & 117 & 136 & 139 \\
\hline $\begin{array}{l}\text { Linear flow } \\
\text { change }\end{array}$ & -0.115 & -0.152 & -0.058 & -0.074 & -0.032 & -0.014 \\
Calibre change & -0.002 & -0.049 & -0.057 & -0.138 & -0.041 & -0.046 \\
\hline
\end{tabular}

Table 3 Mean changes in stem and photocoagulation vessels, experiments 113,115,116,117, and 136

\begin{tabular}{lcccc} 
& \multicolumn{2}{c}{$\begin{array}{l}\text { Stem vessels } \\
\text { Log unit }\end{array}$} & \multicolumn{2}{c}{ Photocoagulation } \\
& $-0.17^{*}$ & 68 & $-0.38^{*}$ & 42 \\
\hline Linear flow & $-0.05^{*}$ & 89 & -0.11 & 78 \\
Calibre & & & & \\
$\begin{array}{l}\text { Volume flow per unit } \\
\quad \text { area }\end{array}$ & $-0.28 \dagger$ & 52 & $-0.60^{*}$ & 25 \\
\hline
\end{tabular}

$\%$ : Terminal value expressed as $\%$ of initial value $*$, †: Significant at $5 \%$ or $1 \%$ level (paired $t$ test) 
BRANCH VESSELS

The mean values for volume inflow per unit area in branch vessels, stem vessels, and superior (control) vessels in the terminal experiments are recorded in Table 4, a series of comparisons ( $t$ test for difference of means) reveals no significant differences, though inspection shows considerably lower values for the stem vessels. When the changes in linear flow, calibre, and $V_{\text {aii }}$ between the initial and terminal experiments expressed in log units are examined and compared to the the corresponding changes in stem and superior vessels significant differences are found ( $t$ test of differences of means) in linear flow and $V_{\text {aii }}$, in respect of the stem vessels, and in calibre (occlusion photocoagulation only) and $V_{\text {aii }}$ in respect of superior vessels. These figures are summarised in Table 5. Investigation of the correlations between different values shows significant regressions of changes in branch $\mathrm{V}_{\mathrm{aii}}$ on changes in branch calibre, but not of changes in branch $V_{\text {aii }}$ on changes in branch linear flow; a negative correlation of changes in branch linear flow on changes in branch calibre; a positive correlation of changes in branch linear flow on changes in stem linear flow, but none of changes in branch calibre on changes in stem linear flow. These results are summarised in Table 6.

\section{SUMMAR Y}

The principal effects of photocoagulation on the

Table 4 Mean volume inflow per unit area, terminal experiments $\left(\mu \mathrm{l} / \mathrm{mm}^{2} / \mathrm{min} \times 10^{2}\right)$. Experiments 113,115 , $116,117,136$, and 139

\begin{tabular}{lllc}
\hline & \multicolumn{3}{c}{ Group } \\
\cline { 2 - 4 } & $\begin{array}{l}\text { Diffuse } \\
\text { photocoagulation }\end{array}$ & Occlusion & Both \\
\hline Superior vessels & 15.4 & 11.4 & 15.0 \\
Branch vessels & 12.6 & 14.9 & 13.9 \\
Stem vessels & 8.1 & 9.3 & 8.6 \\
\hline
\end{tabular}

Table 6 Correlations in branch vessel flow change. Log units. Experiments 113, 115, 116, 117, 136, and 139

\begin{tabular}{llll}
\hline$y$ & $x$ & $r$ & Regression equation \\
\hline Branch $\mathrm{V}_{\text {aii }}$ & Branch calibre & $+0.66^{*}$ & $\mathrm{y}=0.023+0.98 \mathrm{x}$ \\
Branch $\mathrm{V}_{\mathrm{aii}}$ & Branch linear flow +0.12 & \\
Branch linear flow & Branch calibre & $-0.67 *$ & $\mathrm{y}=0.025-1.02 \mathrm{x}$ \\
Branch linear flow & Stem linear flow & $+0.65 *$ & $\mathrm{y}=0.150+0.90 \mathrm{x}$ \\
Branch calibre & Stem linear flow & -0.32 & \\
\hline
\end{tabular}

"Coefficient significant at $5 \%$ level

distribution of retinal blood flow can be summarised.

(1) Superior vessels in the treated eye are admissible for use as controls. A definite calibre reduction occurred between initial and terminal experiments.

(2) Stem vessels show a reduction, principally of linear flow and to a less extent of calibre, proportional to the area reduction following diffuse photocoagulation.

(3) Branch vessels show a slight increase in $V_{\text {aii }}$ after treatment, differing significantly from the changes in stem and superior volume flow.

(4) In the terminal experiments, the observed $V_{a i i}$ is not significantly different in any group of vessels, though the mean values are lower in stem vessels.

\section{Discussion}

The results of this study suggest that after diffuse photocoagulation there is a redistribution of retinal blood flow determined by the reduction of the perfused capillary bed, which increases the total peripheral resistance and results primarily in a passive reduction of linear flow in the stem vessels, although there is also some reduction of calibre. Branch vessels probably experience a slight enhancement of blood flow as shown by the upward trend in $\log$

Table 5 Significant mean changes in branch, superior, and stem vessels. Log units. Experiments 113, 115, 116, 117, 136 , and 139

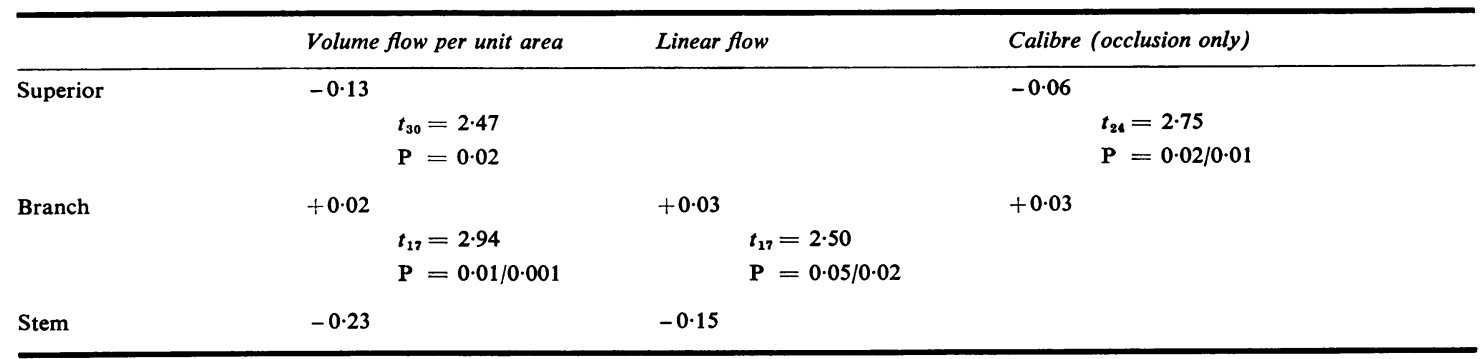


difference values (between initial and terminal experiments), significantly different from both superior (control) and stem vessel values. But this enhancement of flow is not great enough to provide significantly different mean $\mathrm{V}_{\mathrm{aii}}$ values in the terminal experiments when compared to the mean values for superior and stem vessels.

With a few exceptions the redistribution effects resulting from partial or complete occlusion of a small arteriole seem similar to those of diffuse photocoagulation. In this study it has often been necessary to combine the data for statistical treatment.

There is some evidence for homoeostatic control in the branch vessels, in that calibre change and linear flow change are negatively correlated while changes in the volume flow per unit area (a derived value) are correlated only with calibre change. This suggests that calibre change is the determining element in branch vessels in contrast to stem vessels, where the principal change is in linear flow. There is already evidence for homoeostasis in the retina in the work of Dobree (1956), ffytche et al. (1974), and Riva and Loebl (1977).

The purpose of these studies was to obtain an empirical answer to the queries raised in relation to the clinical practice of panretinal photocoagulation. Interpretation must be cautious, because of the healthy status of these tissues as compared to the diseased state in clinical practice and because of the possibility that species differences may invalidate the conclusions. It seems that in healthy tissues much of the tendency to increase branch volume flow may be counteracted by local homoeostatic reaction. Whether this applies to the diseased circulation in retinal vascular disorders requires investigation.

This work was supported by a research grant from the British Diabetic Association, and apparatus was provided by a grant from the Royal National Institute for the Blind.

\section{References}

Dobree, J. H. (1956). Calibre Changes in retinal vessels occurring in raised ocular tension; Circulatory compensation in chronic glaucoma. British Journal of Ophthalmology, 40, 1-13.

ffytche, T. J., Bulpitt, C. J., Kohner, E. M., and Dollery, C. T. (1974). Effect of changes in intraocular pressure on retinal microcirculation. British Journal of Ophthalmology, 58, 514-522.

Goel, B. S. (1974). Arteriolar calibre and areas of supply in the cat retina. Ophthalmic Research, 6, 328-337.

Hill, D. W. (1977). The regional distribution of retinal circulation. Annals of the Royal College of Surgeons of England, 59, 470-475.

Hill, D. W., and Young, S. (1976). Arterial inflow studies of the cat retina, using high-speed cine-angiography. Experimental Eye Research, 23, 35-45.

Laatikainen, L. T. (1976). Regional blood flow in the cat retina. Experimental Eye Research, 23, 47-56.

Meyer-Schwickerath, R. E., and Schott, K. (1968). Diabetic retinopathy and photocoagulation. American Journal of Ophthalmology, 66, 597-603.

Riva, C. E., and Loebl, M. (1977). Auto regulation of blood flow in capillaries of the human macula. Investigative Ophthalmology and Visual Science, 16, 568-571. 